\title{
Research on the New Rural Construction and Traditional Village Protection Based on Evolutionary Game Theory
}

\author{
Jing Wang, Xiaozhou Liu \\ Chengdu Agricultural College \\ Chengdu, Sichuan, China
}

\author{
Zhenyu Zhou \\ Sichuan Jingzheng Construction \& Supervision Service Co,. \\ Ltd. \\ Chengdu, Sichuan, China
}

\begin{abstract}
By investigating the current situation of traditional villages which have been listed in the list of traditional villages, and analyzing the application conditions of evolutionary game between new rural construction and traditional village protection, the evolutionary game model is constructed. Based on the evolutionary game equilibrium theory, the process of evolutionary game is analyzed from the perspective of the new rural construction leader and the traditional village protection leader. Finally, the equilibrium results of the new rural construction leader and the traditional village protection leader on the basis of evolutionary game are obtained, which plays a guiding role in the new rural construction and the traditional village protection.
\end{abstract}

Keywords-Evolutionary Game Theory; New rural construction; Traditional village protection

\section{INTRODUCTION}

The construction of new countryside in China has been put forward since 1950s. The construction of new countryside is an important historical task of building a well-off society and a socialist modern country in an all-round way. It is the master of the work of "agriculture, countryside and farmers" in the new era. It is the product of conforming to the law of modernization, the changing characteristics of urban-rural relations and the yearning for a better life of hundreds of millions of farmers. ${ }^{[1]}$ Chinese civilization is rooted in farming culture. Village is the basic carrier of Chinese civilization. Traditional villages are the living cultural heritage and potential tourism resources. Each traditional village has its unique cultural connotation. ${ }^{[2]}$ In reality, villagers are full of yearning for high-rise buildings and urban life. People hope to live a modern life and enjoy modern civilization. They blindly tear down the old and build new ones, build antique buildings, or simply separate the protection of traditional villages from the natural environment and residents' lives. Although some traditional villages have been preserved, the aborigines of the villages are losing, hollowing out and commercializing seriously. ${ }^{[3]}$ The drawbacks brought about by this form are increasingly apparent, which makes the advantages of new rural construction and traditional village protection not maximized, and neither side will get the greatest benefits.

Evolutionary game theory is one of the most important research fields of modern game theory. It is widely used in the

Scientific research project: College-level scientific research project of Chengdu Agricultural College (cny17-13). fields of ecology, economics, psychology and sociology to study the evolution process and results of group behavior. ${ }^{[4]}$

The core concepts of evolutionary game theory are Evolutionary Stable Strategy (ESS) and Replicator Dynamics (RD). Evolutionary stabilization strategy represents a stable state in which a population resists mutation strategy. The replicated dynamic equation represents the dynamic differential equation describing the frequency or frequency of a population adopting a particular strategy. ${ }^{[5][6]}$

Therefore, this paper uses evolutionary game theory to explore the stable state of the leading Party of new rural construction and traditional village protection under limited rational conditions.

\section{EVOLUTIONARY GAME ANALYSIS OF NEW RURAL} CONSTRUCTION AND TRADITIONAL VILLAGE PROTECTION

Through the investigation in the early stage, it was found that Fuhu Village, Yangma Town, Chongzhou City, Chengdu, and Chuanjiang Village, Lianshan Town, Guanghan City, Deyang City only protected traditional villages; Shanghe Community, Yongsheng Town, Wenjiang District, Jinxiang Village, Wufeng Town, Jintang County, Huaqiu Village, Pingle Town, Qionglai City, Chengdu City, Yuwang Community, Pingluo Town, Qionglai City, Chengdu City, Hongdou Village, Shigu Town, Shifang City, Deyang City, etc. protect and utilize traditional villages; Dayi County Anren Town Street Community, Jintang County Wufeng Town Wufengxi Community and Chengdu Longquanyi District Luodai Town Old Street Community protect and utilize traditional villages and carry out new rural construction. Hence, the hypothetical conditions for the construction of evolutionary game model will be formed.

\section{A. Game Conditions and Parametric Relations}

In order to facilitate the establishment of game model and the analysis of game equilibrium results, the hypothetical conditions of game are listed here.

1. In the process of the game between new rural construction and traditional village protection, participants are divided into two categories: the new rural construction leading Party and the traditional village protection leading party. 
2. The choice of any leading Party in the construction of new countryside includes construction and non-construction. The choice of any leading Party in the protection of traditional villages is based on protection and only for protection. Therefore, the game plan is (construction, protection and utilization), (construction, only protection), (no construction, protection and utilization), (no construction, only protection).

3. When choosing the strategies (construction, protection and utilization) between the leading Party of new rural construction and the leading Party of traditional village protection: the cost paid by the leading Party in the construction of new countryside is $a$; The benefits of the traditional village protection leader are $\mathrm{B}$ and the cost is $b$. $E$ is the choice of construction for the leading Party of new rural construction, while the traditional village protection leading Party chooses the additional value of protection and utilization, mainly for government subsidies and preferential policies. $(E>0, a>0, B>b>0)$

4. When choosing (construction, protection only) strategies: the cost of the construction of the new rural construction leading Party is $a$; the cost paid by the leading Party of traditional village protection is $b$.
5. When choosing (no construction, protection and utilization) strategies: the benefits of traditional village protection leaders are $\mathrm{B}$, and the costs are $b$.

6. When choosing (no construction, only protection) strategy: the cost of the traditional village protection leader is $b$.

7. Probability: Expressing the Probability of Leading Party's Choice of "construction" Strategy in New Rural Construction with $p$ AND $1>p>0$, then the probability of choosing "no construction" strategy is $1-p$; At the same time, $q$ is used to express the probability that the traditional village protection leader chooses the strategy of "protection and utilization" and $1>q>0$, then the probability of choosing a "protection-only" strategy is $1-q$.

\section{B. Constructing Evolutionary Game Model}

From the above game conditions, we can get the income matrix as shown in TABLE I.

TABLE I.

GAME INCOME MATRIX

\begin{tabular}{|c|c|c|c|}
\hline & \multicolumn{2}{|c|}{ The Leading Party of Traditional Village Protection } \\
\hline & & $\begin{array}{l}\text { Protection and } \\
\text { Utilization }(q)\end{array}$ & protection-only $(1-q)$ \\
\hline \multirow{2}{*}{ Leading Party of New Rural Construction } & Construction $(p)$ & $-a+E, B-b+E$ & $-a,-b$ \\
\hline & No construction $(1-p)$ & $0, B-b$ & $0,-b$ \\
\hline
\end{tabular}

\section{Evolutionary Game Analysis}

1) Benefits of both the new rural construction leader and the traditional village protection leader

The expected benefits of "construction" and "no construction" chosen by the leading Party in the construction of new countryside $U_{1} U_{2}$ and the average benefit of the leading

group in the construction of New Countryside $\bar{U}$ is respectively:

$$
\begin{gathered}
U_{1}=q(-a+E)+(1-q)(-a)=q E-a \\
U_{2}=0 \\
\bar{U}=p U_{1}+(1-p) U_{2}=p(q E-a)
\end{gathered}
$$

The expected benefits of the traditional village protection leading Party choosing "protection and utilization" and "protection only" $V_{1} 、 V_{2}$ and the average benefit of the

dominant group in traditional village protection $\bar{V}$ is respectively:

$$
\begin{gathered}
V_{1}=p(B-b+E)+(1-p)(B-b)=p E+B-b \\
V_{2}=p(-b)+(1-p)(-b)=-b
\end{gathered}
$$

$$
\bar{V}=q V_{1}+(1-q) V_{2}=q(p E+B-b)+(1-q)(-b)
$$

2) Duplicate dynamic side of leading group in new rural construction and traditional village protection leading group

The replication dynamic analysis of the new rural construction leading group and the traditional village protection leading group is carried out respectively, and the replication dynamic equation of the new rural construction leading Party choosing the "construction" strategy is obtained as follows:

$$
F(p)=d_{p} / d_{t}=p\left(U_{1}-\bar{U}\right)
$$

This replication dynamic equation describes the evolutionary process of the population ready to choose strategy $p$ and strategy $1-p$, which reflects the basic idea in the process of replication dynamics: if the result of strategy $p$ is better than the average level, the proportion of the population choosing strategy $p$ will increase. By substituting the formulas (1) and (3) into the equation of replication dynamics (7), we can obtain:

$$
F(p)=d_{p} / d_{t}=p[q E-a-p(q E-a)]=p(1-p)(q E-a)
$$

Similarly, the replication dynamic equation of the traditional village protection leader choosing the strategy of "protection and utilization" can be obtained as follows: 


$$
F(q)=d_{q} / d_{t}=q\left(V_{1}-\bar{V}\right)
$$

By substituting the formulas (4) and (6) into the equation of replication dynamics (9), we can obtain:

$$
\begin{aligned}
& F(q)=d_{q} / d_{t} \\
& =q\{(p E+B-b)-[q(p E+B-b)+(1-q)(-b)]\} \\
& =q\{(1-q)(p E+B-b)-(1-q)(-b)\} \\
& =q(1-q)(p E+B)
\end{aligned}
$$

3) Analysis of replication dynamic equation (8) based on the leading group of new rural construction

When $q E-a=0$, that is when $q=\frac{a}{E}, d_{p} / d_{t}$ is always equals to 0 ,that is for $p$, everything is stable. Its practical significance is: when the leading Party of new rural construction chooses the proportion of "construction" is $\frac{a}{E}$, there is no difference between the two strategies of "protection and utilization" and "protection only" adopted by the leading Party of traditional village protection, as showed in Fig. 1.

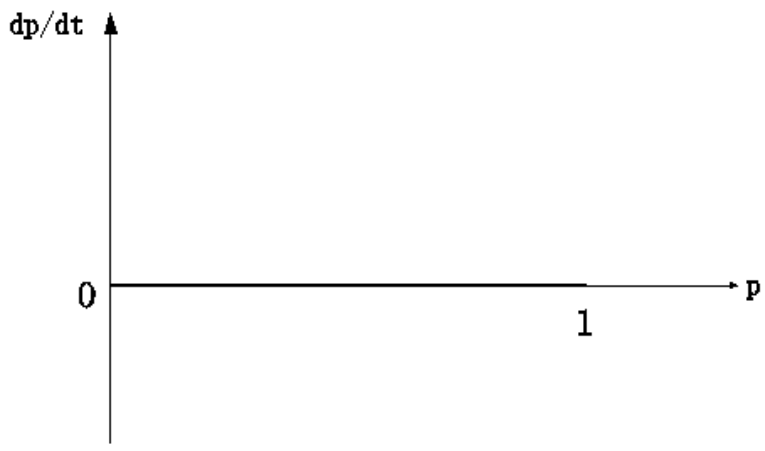

Fig. 1 No difference in protection utilization decisions

When $q\left\langle\frac{a}{E}, U_{1}-\bar{U}\left\langle 0, p^{*}=0\right.\right.$ and $p^{*}=1$ is two stable state of $p$. The expected benefit of selecting "protection and utilization" is less than the average benefit. So $p^{*}=0$ is an evolutionary stabilization strategy in replication dynamics. Because in the state of $p^{*}=1$, once the "only protection" strategy is adopted, the dominant group of traditional village protection will learn by trial and error, and then adopt the "only protection" strategy. While in the state of $p^{*}=0$, it is stable to a small number of errors. It can not only resist the invasion of mutation, but also achieve stability in the adjustment of dynamic strategy. Hence, $p^{*}=0$ is an evolutionary stabilization strategy, that is, to choose the "only protection" strategy, as showed in Fig. 2

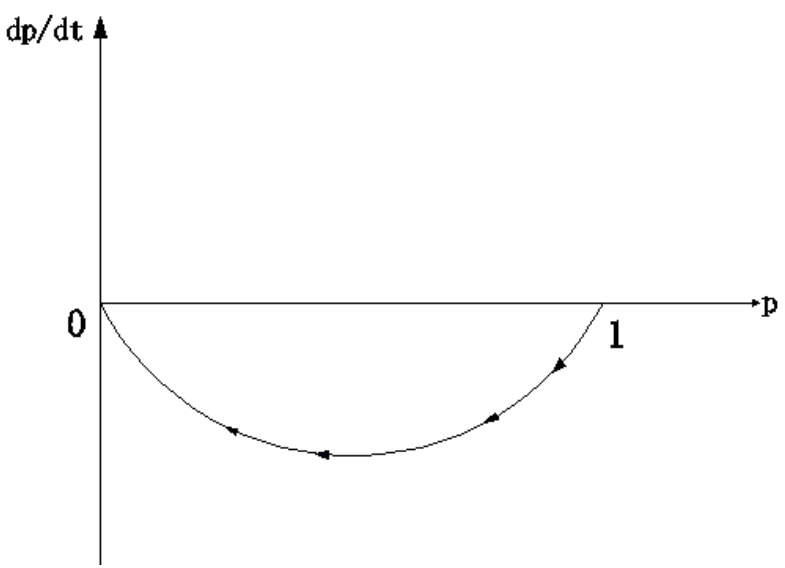

Fig. 2 Selecting the "Protect Only" Policy

When $q>\frac{a}{E}, U_{1}-\bar{U}>0$. At this point, $p^{*}=1$ is an evolutionary stabilization strategy under replication dynamics, that is, to choose the "protection and utilization" strategy, as showed in Fig. 3

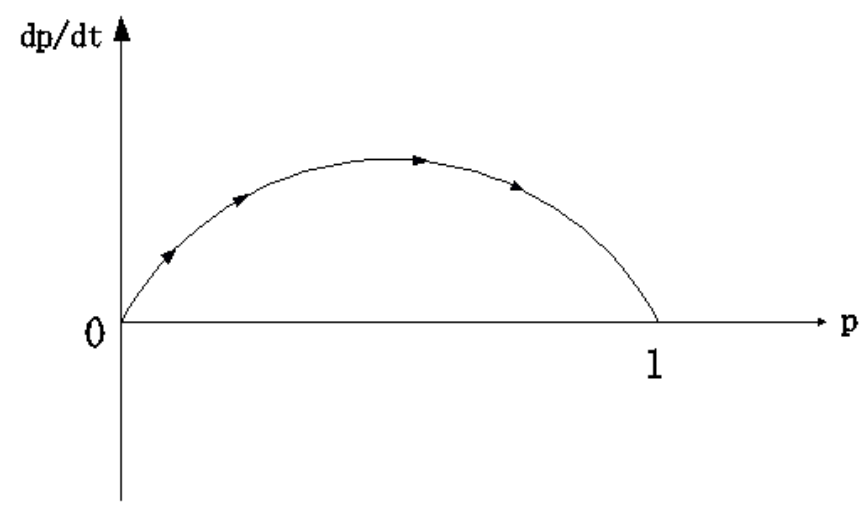

Fig. 3 Selection of "Protection and Utilization" Strategy

In summary, the demarcation point between the two evolutionary stabilization strategies $p^{*}=0$ and $p^{*}=1$ is $q=\frac{a}{E}$. It can be seen that the smaller the cost , the greater the additional value $E$, and the greater the driving force of the new rural construction leading party.

4) Analysis of replication dynamic equation (10) based on the dominant group of traditional village protection

Since $p E+B>0, q^{*}=0$ and $q^{*}=1$ is two stable state of $q$. There is no difference between the two strategies of "construction" and "non-construction", as showed in Fig. 4. 
$\mathrm{dq} / \mathrm{dt} /$

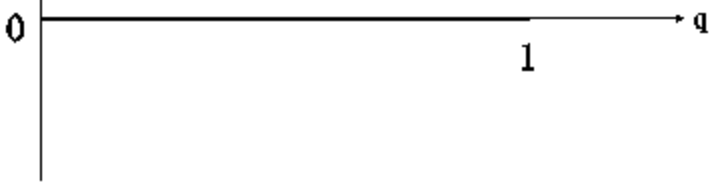

Fig. 4 No difference in construction decision-making

$$
\begin{aligned}
& F^{\prime}(q)=(1-2 q)(p E+B) \\
& \because p E+B>0,0<q<1 \\
& \therefore F^{\prime}(0)>0, \quad F^{\prime}(1)<0
\end{aligned}
$$

So $q^{*}=1$ is an evolutionary stabilization strategy, that is, the dominant party of traditional village protection chooses "protection and utilization".

\section{Results}

In summary, the demarcation point of evolutionary stabilization strategy $p^{*}=0$ and $p^{*}=1$ is $q=\frac{a}{E}$. The cost $a$ of the new rural construction leading Party is reduced, the additional value $E$ is increased, and the driving force of the new rural construction leading Party "construction" is greater. While $q^{*}=1$ is an evolutionary stabilization strategy, that is to say, the dominant party of traditional village protection chooses "protection and utilization".

Therefore, the new rural construction leader and the traditional village protection leader will eventually tend to choose the evolutionary stable strategy (construction, protection and utilization).

\section{Practical SignificANCE}

The traditional villages in this investigation and study are listed in the list of traditional villages, which have been protected. But in reality, due to the lack of protection consciousness and other reasons, a large number of traditional villages disappeared rapidly, which destroyed the crystallization of farming culture in China for thousands of years.

In addition, these villages listed in the list of traditional villages have tourism value overnight. Most of the traditional villages have regarded tourism as a way to get rid of poverty and become rich. In order to pursue economic interests, blind over-exploitation has caused new damage to traditional villages, and uniform model development may cause destructive impact on traditional villages that have been listed in the list of traditional villages. For this reason, traditional villages such as Shanghe Community, Yongsheng Town, Wenjiang District, Jinxiang Village, Wufeng Town, Jintang County, Huacai Village, Pingle Town, Qionglai City, Chengdu, all retain dilapidated houses to the greatest extent and retain their original functions and layout. On the basis of the original traditional buildings, tourist attractions should be built, tourist service reception capacity should be improved, and villagers' income should be increased.

At the same time, with the acceleration of modernization and the construction of new countryside, many aborigines of traditional villages go out to work or move out of villages, which make the hollowing of traditional villages more and more serious. But this is not enough. For the sake of tourism, some traditional villages even move the aborigines out as a whole. Little do we know that in this way, culture and customs have also moved away, and it is no longer a traditional village. The protection of traditional villages is not only to protect material culture, but also to protect intangible culture. Therefore, new countryside has been built or is under construction in Anren Street Community of Dayi County, Wufeng Street Community of Wufeng Town of Jintang County and Luodai Town of Longquanyi District of Chengdu City. After the aborigines are moved out nearby, the cultural and historical values of traditional villages are excavated. The use of tourism resources to solve the employment of villagers, but also let villagers more love their own villages, loves their own culture.

According to the investigation and evolutionary game analysis, we have come to the conclusion (construction, protection and utilization). In addition, the formulation of laws and regulations for the protection of traditional villages, the scientific planning of the protection of traditional villages in the process of new rural construction, the excavation and inheritance of the material and intangible culture of traditional villages, the rational utilization of tourism resources, and the active participation of villagers are also needed to make the protection of traditional villages sustainable development.

\section{REFERENCES}

[1] The Central Committee of the Communist Party of China, the State Council, the Central Committee of the Communist Party of China and the State Council on the implementation of the rural revitalization strategy [Z].2018-01-02.

[2] Central Rural Work Leading Group Office. Rural Revitalization Strategic Plan [Z].2018-02-05.

[3] Feng Yicai. Cultural empty nest and its countermeasures [N]. Wen Wei Po, 2007-03-12.

[4] Xie Zhiyu. Evolutionary Game Theory under the Condition of Bounded Rationality[J].Journal of Shanghai University of Finance and Economics,2001,5:3-9.

[5] Xie Zhiyu. Economic Game Theory (Second Edition) [M]. Fudan University Press, 2002, 233-275.

[6] GILBOA I, MATSUI A. Society and equilibrium [J]. Econometrica, 1991, 59 (3): 859-867. 